Check for updates

Cite this: RSC Adv., 2018, 8, 21651

\title{
Hybrid films with excellent oxygen and water vapor barrier properties as efficient anticorrosive coatings $\dagger$
}

\author{
Jiajie Wang, Ting Pan, Jian Zhang, Xiaozhi Xu, Qing Yin, Jingbin Han (D)* \\ and Min Wei iD
}

\begin{abstract}
Gas and moisture barrier materials are of crucial importance in various application fields, including food/ drug packaging and encapsulation of electronic devices. Herein, a dual-barrier film to gas and water vapor was fabricated by a facile and cost-effective spin-coating of amphiphilic surfactant (Tween 80 ) modified LDH nanoplatelets (denoted as LDH-80) and polydimethylsiloxane (PDMS). The resultant (LDH80/PDMS) $)_{15}$ film exhibits low $\mathrm{O}_{2}$ and $\mathrm{H}_{2} \mathrm{O}$ transmission rates with $\sim 0.701$ and $\sim 0.049 \mathrm{~cm}^{3} \mathrm{~m}^{-2} \mathrm{~d}^{-1}$ $\mathrm{atm}^{-1}$, respectively, smaller than those for most of the reported barrier materials. The remarkable barrier properties are ascribed to the prolonged diffusion length for gas permeation and improved inorganicorganic interfacial compatibility between LDH-80 and PDMS. Taking advantage of this unique dualbarrier property, an aluminum foil substrate coated with (LDH-80/PDMS) ${ }_{n}$ film displays an excellent anticorrosion effect due to the inhibition of oxygen-consuming corrosion, which enables the (LDH-80/ $\mathrm{PDMS}_{n}$ films to be promising candidates in metal surface protection.
\end{abstract}

Received 4th May 2018

Accepted 7th June 2018

DOI: $10.1039 / \mathrm{c} 8 \mathrm{ra0} 8819 \mathrm{~h}$

rsc.li/rsc-advances drug delivery, energy storage and smart response. ${ }^{24-31}$ Our recent work reported the design and assembly of $\mathrm{LDH} /$ polymer films as effective barrier materials toward $\mathrm{O}_{2}, \mathrm{~N}_{2}, \mathrm{CO}_{2}$ and $\mathrm{He}$ gas. $^{32-35}$ Their barrier property is ascribed to the prolonged diffusion pathway of gas molecules with the introduction of LDH nanosheets, and the decreased free volume by the modification of organic-inorganic interface. However, upon long-term exposure in moisture atmosphere, their gas barrier property will be damaged. The hydrophilic characteristic of LDHs and polymers enables the adsorption/diffusion of water molecule into films, resulting in the plasticization of polymer matrix and finally deterioration of the gas barrier property. To solve this problem, a dual-barrier film toward gas and water vapor by assembling surfactant-modified LDH with a hydrophobic polymer would be a promising candidate.

In this work, an organic-inorganic composite film was fabricated via alternate spin-coating technique, consisting of an amphiphilic surfactant (Tween 80) modified LDH nanoplatelets (denoted as LDH-80) and polydimethylsiloxane (PDMS) as building blocks (Scheme 1). The resulting (LDH-80/PDMS $)_{n}$ films show dual-barrier behavior toward water and oxygen molecule simultaneously. The combination of oxygen and water dual-barrier performance in one single film has rarely been demonstrated in previous reports. By virtue of such an interesting property, the aluminum foil coated by (LDH-80/PDMS $)_{n}$ film exhibits a suppressed oxygen-consuming corrosion in an oxygen/moisture enriched atmosphere.
State Key Laboratory of Chemical Resource Engineering, Beijing Advanced Innovation Center for Soft Matter Science and Engineering, Beijing University of Chemical Technology, Beijing, 100029, China. E-mail: hanjb@mail.buct.edu.cn; Tel: +86-10-64412131

$\dagger$ Electronic supplementary information (ESI) available. See DOI: 10.1039/c8ra03819h 

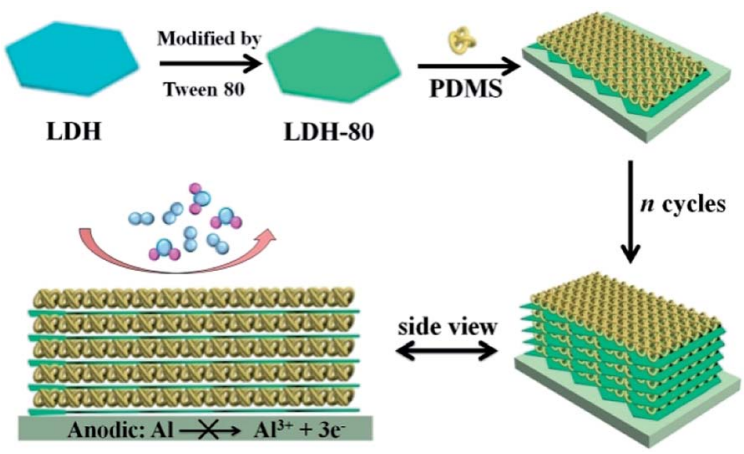

Scheme 1 Schematic illustration for the assembly process of (LDH$80 /$ PDMS $)_{n}$ film on aluminum foil substrate for anti-corrosion protection.

\section{Experimental}

\section{Reagents and materials}

Analytical grade chemicals including $\mathrm{Mg}\left(\mathrm{NO}_{3}\right)_{2} \cdot 6 \mathrm{H}_{2} \mathrm{O}$, $\mathrm{Al}\left(\mathrm{NO}_{3}\right)_{3} \cdot 9 \mathrm{H}_{2} \mathrm{O}, \mathrm{NaOH}$, concentrated sulphuric acid $\left(\mathrm{H}_{2} \mathrm{SO}_{4}\right)$, hydrogen peroxide $\left(\mathrm{H}_{2} \mathrm{O}_{2}\right)$ and polydimethylsiloxane (PDMS) were obtained from Beijing Yili Fine Chemicals Co. Ltd. Tween 80 (Fig. S1, ESI†) was purchased from Aladdin Chemicals Co. Ltd. and used without further purification. Deionized water was used in all the experiments.

\section{Synthesis of MgAl-LDH colloid}

MgAl-LDH colloid was synthesized by the separate nucleation and aging steps method reported by our group. ${ }^{36,37}$ Typically, $100 \mathrm{ml}$ mixed salt solution $\left(\mathrm{Mg}\left(\mathrm{NO}_{3}\right)_{2} \cdot 6 \mathrm{H}_{2} \mathrm{O}: 0.2 \mathrm{M}\right.$ and $\left.\mathrm{Al}\left(\mathrm{NO}_{3}\right)_{3} \cdot 9 \mathrm{H}_{2} \mathrm{O}: 0.1 \mathrm{M}\right)$ and $400 \mathrm{ml} \mathrm{NaOH}$ solution $(0.15 \mathrm{M})$ were simultaneously added to a colloid mill with rotor speed of $3000 \mathrm{rpm}$ and mixed for $1 \mathrm{~min}$. The resulting slurry was obtained via centrifugation and washed several times and then dispersed in $400 \mathrm{ml}$ of deionized water. This aqueous suspension was transferred into a stainless steel autoclave with a Teflon lining. Upon hydrothermal treatment at $110{ }^{\circ} \mathrm{C}$ for $24 \mathrm{~h}$, the LDH colloidal suspension was obtained after removal of the precipitate.

\section{Modification of LDH by Tween 80}

Tween 80 (5 ml) was added to $200 \mathrm{ml} \mathrm{LDH}$ colloidal suspension (solid content: $1.14 \%$ ), followed by stirring vigorously for $4 \mathrm{~h}$ at room temperature. After that, the $\mathrm{LDH}$ nanoplatelet was modified by Tween 80 (defined as LDH-80). Subsequently, the modified LDH was washed thoroughly with water to remove the excess Tween 80 . Finally, the LDH-80 was dispersion in water to form a stable colloidal suspension (solid content: $\sim 1.0 \%$ ) to fabricate (LDH-80/PDMS $)_{n}$ films.

\section{Fabrication of the (LDH-80/PDMS $)_{n}$ films}

The (LDH-80/PDMS $)_{n}$ films were constructed on specific substrates by applying the spin-coating technique. Polyethylene terephthalate (PET) film was used as substrate for oxygen/water vapor transmission rate test; Al foil was employed for the measurement of anticorrosive property; and quartz glass substrate (purity $>99.9 \%$ ) was used for other characterizations. Prior to deposition, the substrates were treated in an ultrasonic bath with deionized water, ethanol and acetone for 10 min sequentially. PDMS was selected due to its hydrophobic characteristic and excellent film-forming property. The LDH-80 colloidal suspension $(\sim 1 \%)$ and pure PDMS liquid without solvent were spin-coated onto a pre-cleaned substrate with $2000 \mathrm{rpm}$ for $60 \mathrm{~s}$ and $5000 \mathrm{rpm}$ for $60 \mathrm{~s}$ alternately. The film was dried at ambient temperature in air for $20 \mathrm{~min}$ after each spin-coating process. Subsequently, a series of these operations for LDH-80 and PDMS were repeated $n$ times to obtain the $(\mathrm{LDH}-80 / \mathrm{PDMS})_{n}$ multilayer films. The initial and terminational layers are LDH-80 and PDMS, respectively. As a comparison sample, the (LDH/PDMS $)_{n}$ films using unmodified LDH were prepared by a similar spin-coating process.

\section{Results and discussion}

\section{Characterization of LDH and LDH-80}

Fig. 1A illustrates the XRD pattern of the MgAl-LDH sample with $2 \theta$ at $10.2^{\circ}, 20.3^{\circ}, 34.9^{\circ}, 38.1^{\circ}$ and $61.0^{\circ}$, which can be indexed as (003), (006), (012), (015) and (110) reflections of a nitrate-LDH phase. No other crystalline phase is detected, indicating the high purity of the product. SEM image of the LDH shows hexagonal plate-like nanocrystals with diameter of $\sim 105 \mathrm{~nm}$ (Fig. 1B). The LDH nanoplatelets were then modified with an amphipathic surfactant (Tween 80) to construct a hydrophobic surface (denoted as LDH-80) for the enhancement of interfacial compatibility with polymer. The XRD pattern of $\mathrm{LDH}-80$ shows a (003) diffraction in the same position as that of pristine $\mathrm{LDH}$, which illustrates the interlayer spacing of $\mathrm{LDH}$ keeps unchanged, indicting the absence of Tween 80 in LDH interlayer. Fig. 1D illustrates LDH-80 nanoplatelets maintain its original plate-like morphology, with a particle size distribution of 75-152 $\mathrm{nm}$ and an average size of $\sim 110 \mathrm{~nm}$ (Fig. S2, ESI $\dagger$ ). In addition, the weight loading of Tween 80 in the LDH-80 hybrid was calculated to be $12.5 \%$ from thermogravimetric analysis (TGA, Fig. S3, ESI $\dagger$ ).

The modification of LDH with Tween 80 was further illustrated by FT-IR spectra. The characteristic FT-IR bands of Tween 80 are assigned as follows (Fig. 2, red curve): $3550 \mathrm{~cm}^{-1}(-\mathrm{OH}$ stretching vibration), $2927 \mathrm{~cm}^{-1}$ (-CH stretching of $-\mathrm{CH}_{3}$ groups), $2852 \mathrm{~cm}^{-1}$ (-CH stretching of $-\mathrm{CH}_{2}$ groups), $1740 \mathrm{~cm}^{-1}$ ( $\mathrm{C}=\mathrm{O}$ stretching) and $1095 \mathrm{~cm}^{-1}$ (C-O-C bonding). For $\mathrm{LDH}$ (black curve), the bands at 3480 and $1630 \mathrm{~cm}^{-1}$ are attributed to the hydroxy $(-\mathrm{OH})$ stretching vibration of its host layer and interlayer crystal water, respectively. Bands at 1380 and $740 \mathrm{~cm}^{-1}$ indicate the presence of $\mathrm{NO}_{3}{ }^{-}$in $\mathrm{LDH}$ gallery. In the case of LDH-80 sample (blue curve), the characteristic peaks of both LDH and Tween 80 are observed. It should be noted that the band of $\mathrm{C}=\mathrm{O}$ stretching vibration for $\mathrm{LDH}-80$ shows a redshift relative to Tween-80 (from $1740 \mathrm{~cm}^{-1}$ to $1660 \mathrm{~cm}^{-1}$ ). In contrast, this band remains unchanged $\left(1740 \mathrm{~cm}^{-1}\right)$ for the physical mixture of $\mathrm{LDH}$ and Tween 80 , the $\mathrm{C}=\mathrm{O}$ stretching vibration appears at $1740 \mathrm{~cm}^{-1}$ (Fig. S4, ESI $\dagger$ ). These results may suggest the presence of interfacial interaction between Tween 80 and LDH. 

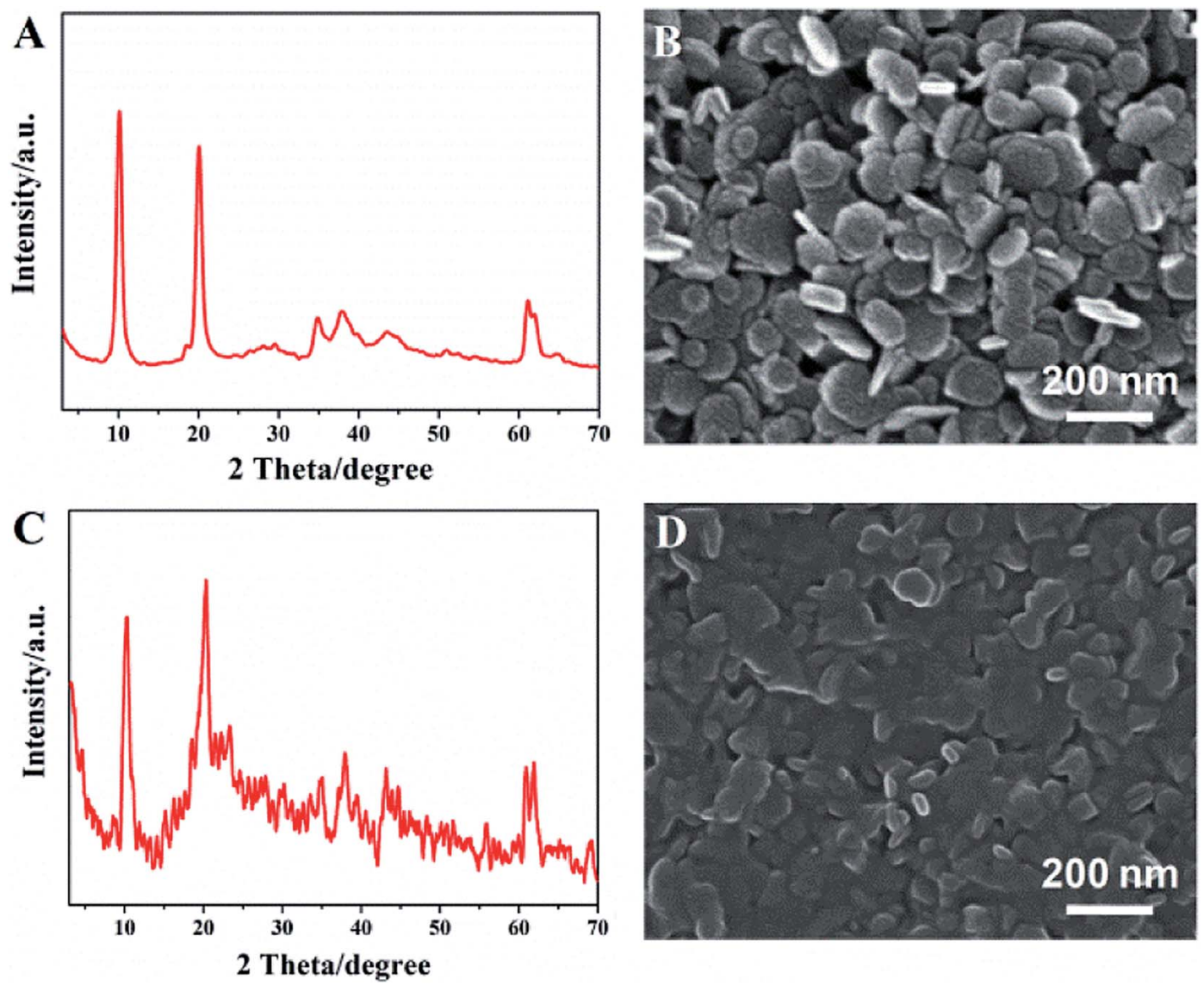

Fig. 1 XRD patterns and SEM images of pristine LDH ( $A$ and B) and Tween 80 modified LDH (C and D).

Structural and morphological analyses of (LDH-80/PDMS) ${ }_{n}$ films

UV-vis absorption spectra of the (LDH-80/PDMS $)_{n}$ films with various bilayer numbers are shown in Fig. 3A. The characteristic absorption intensity of PDMS at $192 \mathrm{~nm}$ enhances linearly with the increase of bilayer number $n$ (Fig. 3A, inset), indicating a stepwise and regular growth process for the (LDH-80/PDMS $)_{n}$

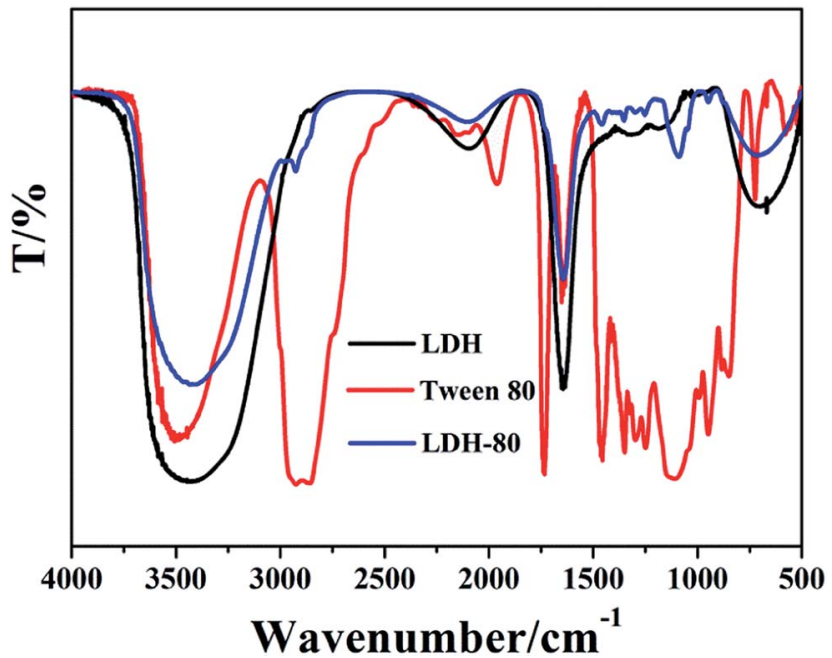

Fig. 2 FT-IR spectra of Tween 80, pristine LDH and LDH-80 samples. films. XRD pattern (Fig. S5, ESI $\dagger$ ) of the obtained (LDH-80/ PDMS $)_{15}$ film displays a peak at $2 \theta=10.2^{\circ}$, which is attributed to the (003) reflection of the $\mathrm{LDH}$ phase.

The top-view SEM image (Fig. 3B) displays the homogeneity of the (LDH-80/PDMS $)_{15}$ film. The thickness of (LDH-80/PDMS $)_{n}$ films with various $n$ can be estimated from their side-view SEM images (inset of Fig. 3B and S6, ESI $\dagger$ ), which increases gradually from 3.47 to $12.95 \mu \mathrm{m}$ as the bilayer number varies from 3 to 15 , indicating an average thickness increment of $0.89 \mu \mathrm{m}$ per bilayer cycle (Fig. S7, ESI $\dagger$ ). Upon scratching of the film surface, no delamination or peeling occurs, demonstrating a strong adhesion of the film to the substrate (Fig. S8, ESI $\dagger$ ). The AFM topographical image $(5 \mu \mathrm{m} \times 5 \mu \mathrm{m})$ of the $(\mathrm{LDH}-80 / \mathrm{PDMS})_{15}$ film along with a respective surface profile is illustrated in Fig. S9 (ESI $\dagger$ ), from which a homogeneous and smooth surface is observed with root mean square roughness $\left(R_{\mathrm{q}}\right)$ of $\sim 10.9 \mathrm{~nm}$. In comparison, the $R_{\mathrm{q}}$ value for (LDH/PDMS $)_{n}$ and pure PDMS film is $\sim 37.6$ and $\sim 7.2 \mathrm{~nm}$, respectively (Fig. S10, ESI $\dagger$ ). The relatively higher surface roughness of $(\mathrm{LDH} / \mathrm{PDMS})_{n}$ film is probably related with its lower interfacial compatibility between LDH and PDMS. In addition, the (LDH/PDMS $)_{15}$ film shows a remarkable optical transparency (Fig. S11, ESI†) with an average transmittance of $\sim 76 \%$ in $400-800 \mathrm{~nm}$.

The arrangement of inorganic lamella is one of the crucial parameters governing the barrier property of organic-inorganic films. The orientation of $\mathrm{LDH}$ platelets in $(\mathrm{LDH}-80 / \mathrm{PDMS})_{n}$ 

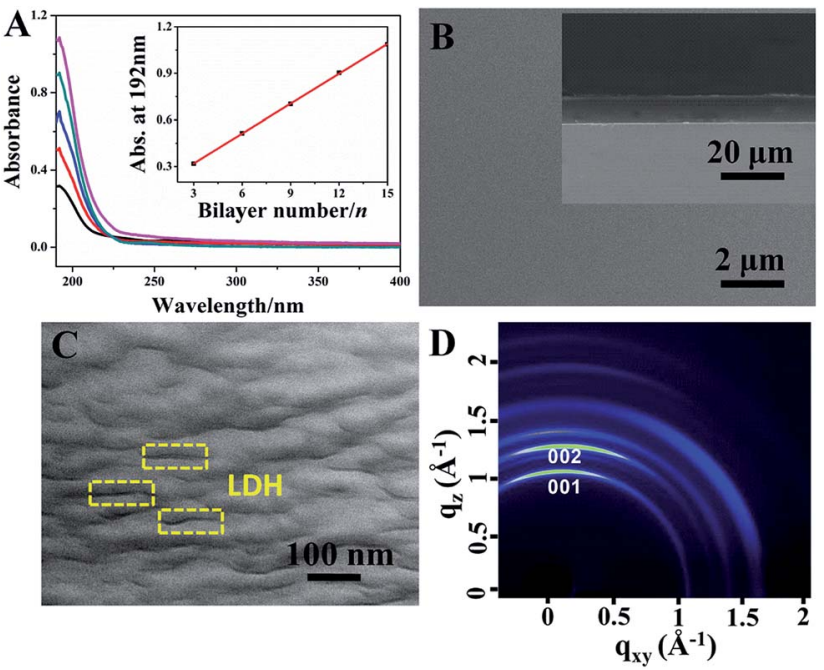

Fig. 3 (A) UV-vis absorption spectra of the (LDH-80/PDMS $)_{n}(n=3-$ 15) films on quartz glass substrates (inset: the linear relationship between absorbance at $192 \mathrm{~nm}$ and bilayer number $n$ ); (B) top-view and side-view (inset) SEM images of (LDH-80/PDMS) ${ }_{15}$ film; (C) sideview TEM image and (D) 2D grazing-incidence XRD patterns for ( $\mathrm{LDH}$ 80/PDMS) ${ }_{15}$ film.

films was observed by TEM using a resin-embedding and section method (see details in the ESI $\dagger$ ), which shows the layers of LDH platelets as some dark lines with a distinct stratified architecture (Fig. 3C), illustrating the good dispersion and high orientation of $\mathrm{LDH}$ platelets in the hybrid films. In addition, the $2 \mathrm{D}$ grazing-incidence XRD pattern of the ( $\mathrm{LDH}-80 / \mathrm{PDMS})_{15}$ film displays increased reflections along the $q_{x y}$ (in-plane) axes (Fig. 3D), further indicating the parallel orientation of $\mathrm{LDH}$ platelets to the substrate.

The surface wettability of a film is closely related with its water molecule permeation property. The water contact angle test was performed to investigate the surface wettability of resultant films. As shown in Fig. 4, the bare substrate, LDH and $(\mathrm{LDH} / \mathrm{PDMS})_{15}$ (fabricated by using unmodified LDH) film are hydrophilic with water contact angle less than $90^{\circ}$. The pure PDMS film shows a slightly hydrophobic feature with water contact angle of $97^{\circ}$. In contrast, the (LDH-80/PDMS $)_{15}$ film exhibits a water contact angle of $115^{\circ}$, indicative of a more

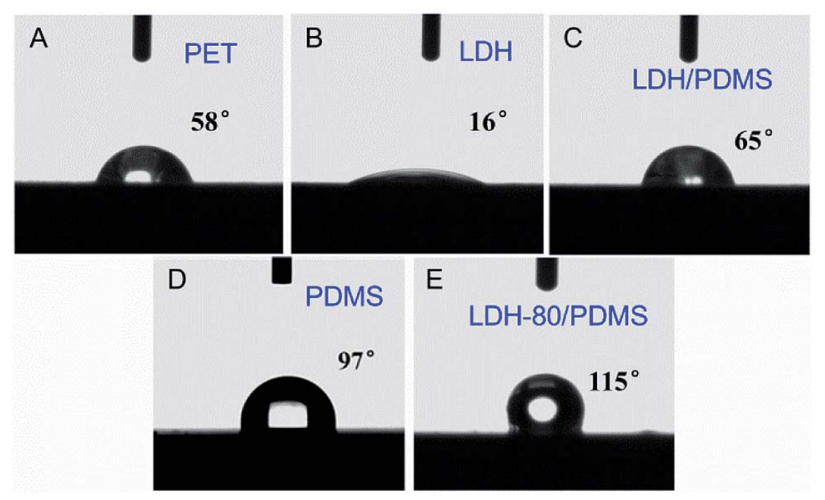

Fig. 4 Water contact angle measurements on (A) bare PET, (B) pristine $\mathrm{LDH},(\mathrm{C})(\mathrm{LDH} / \mathrm{PDMS})_{15}$, (D) pure PDMS and (E) (LDH-80/PDMS) ${ }_{15}$ film. hydrophobic surface. The hydrophobic surface of (LDH-80/ PDMS $)_{15}$ coating would facilitate the improvement of water vapor barrier property.

\section{Oxygen/water vapor dual-barrier performance of (LDH-80/ PDMS $)_{n}$ films}

The oxygen transmission rate (OTR) and water vapor transmission rate (WVTR) were measured to investigate the barrier property of (LDH-80/PDMS $)_{n}$ films on PET substrate (Fig. 5). The pristine PET film displays OTR and WVTR of $\sim 111.271$ and $\sim 31.788 \mathrm{~cm}^{3} \mathrm{~m}^{-2} \mathrm{~d}^{-1}$ atm ${ }^{-1}$, respectively. The coating of (LDH80/PDMS $)_{n}$ films leads to dramatic decrease in OTR and WVTR. It is observed that the OTR values for (LDH-80/PDMS $)_{n}$ films reduce from $\sim 68.589$ to $\sim 0.701 \mathrm{~cm}^{3} \mathrm{~m}^{-2} \mathrm{~d}^{-1} \mathrm{~atm}^{-1}$; and the WVTR values decrease from $\sim 12.153$ to $\sim 0.049 \mathrm{~cm}^{3} \mathrm{~m}^{-2} \mathrm{~d}^{-1}$ $\mathrm{atm}^{-1}$ as the bilayer number $n$ increases from 5 to 15 . In contrast, the pure PDMS film with the same thickness as (LDH80/PDMS $)_{15}$ film displays a relatively weak barrier ability with OTR of $\sim 106.032 \mathrm{~cm}^{3} \mathrm{~m}^{-2} \mathrm{~d}^{-1}$ atm ${ }^{-1}$ and an unexceptional reduction of WVTR to $\sim 18.492 \mathrm{~cm}^{3} \mathrm{~m}^{-2} \mathrm{~d}^{-1}$ atm ${ }^{-1}$ (Fig. S12, ESI $\dagger$ ). In addition, the (LDH/PDMS $)_{15}$ film exhibits higher OTR and WVTR values compared with (LDH-80/PDMS) ${ }_{15}$ film, which is ascribed to the weak interfacial compatibility between $\mathrm{LDH}$ and PDMS matrix. The results illustrate the incorporation of highly-oriented LDH-80 nanoplates suppresses the diffusion of $\mathrm{O}_{2}$ and $\mathrm{H}_{2} \mathrm{O}$ molecules as a result of the increased diffusion length; the hydrophobicity of the (LDH-80/PDMS $)_{15}$ film further prohibits the adsorption of water molecule to the film surface and hence an enhanced water vapor barrier property is achieved. Compared with other reported barrier materials, the oxygen and water vapor barrier properties of (LDH-80/PDMS $)_{15}$ films is among the highest level (Table S1, ESI $\dagger$ ). And the dualbarrier performance of (LDH-80/PDMS $)_{15}$ film would be sufficient for food packaging and encapsulation of electronic devices, such as LCD/LED display and photovoltaic module (Fig. S13, ESI $\dagger$ ).

\section{The anti-corrosion property of (LDH-80/PDMS $)_{n}$ films}

Base on the unique dual-barrier property of the (LDH-80/ PDMS $)_{n}$ films toward oxygen and water vapor, it is expected that

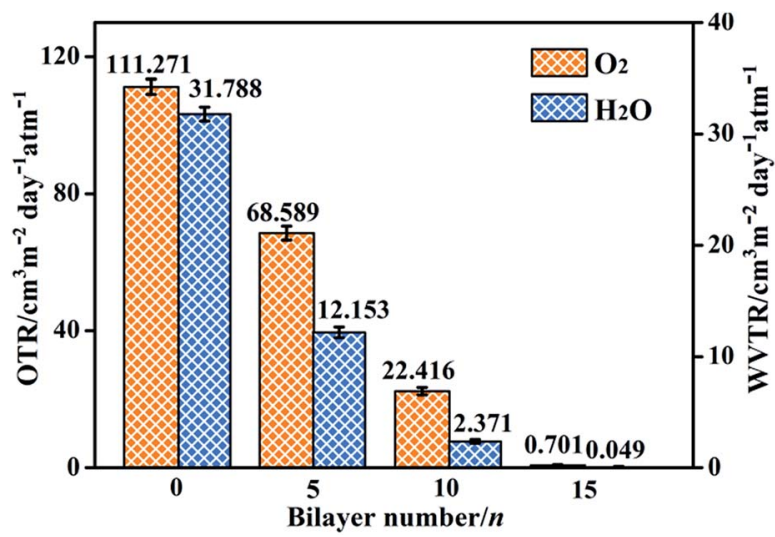

Fig. 5 OTR and WVTR values of bare PET substrate $(n=0)$ and (LDH$80 /$ PDMS $)_{n}$ films with $n=5,10$ and 15 , respectively. 
such films may impose anti-corrosion effect on the metal protection, as the metallic corrosion is induced by the oxidation under oxygen and moisture-enriched conditions. The (LDH-80/ PDMS $)_{n}$ films were assembled on $\mathrm{Al}$ foil substrate to study the anti-corrosion effect by examining their electrochemical impedance spectroscopy (EIS) and polarization curves in $3.5 \mathrm{wt} \% \mathrm{NaCl}$ aqueous solution. The Nyquist plots of (LDH-80/ PDMS)n films are presented in Fig. 6A. It is observed that the $(\mathrm{LDH}-80 / \mathrm{PDMS})_{n}$ films exhibit an increment in the order of magnitudes for interfacial electron-transfer resistance upon increasing bilayer number from 5 to 15 , indicating an improved anti-corrosion behavior. Moreover, compared with the bare $\mathrm{Al}$ substrate, PDMS and (LDH/PDMS) ${ }_{15}$ films, the (LDH-80/ PDMS $)_{15}$ film displays the largest electron-transfer resistance (Fig. 6B), indicative of the best anti-corrosion property, which is ascribed to its superior oxygen and water molecule barrier behavior.

Tafel extrapolation measurements (Fig. 7A) were carried out to give a further understanding on the metallic protection by (LDH-80/PDMS $)_{n}$ films. The bare Al substrate shows a corrosion potential $\left(E_{\text {corr }}\right)$ of $-1418 \mathrm{mV}$; while the $E_{\text {corr }}$ of (LDH-80/PDMS $)_{n}$ coated $\mathrm{Al}$ exhibits a positive shift from -1276 to $-672 \mathrm{mV}$ as $n$ increases from 5 to 15 . The corrosive current density $\left(I_{\text {corr }}\right)$ of the (LDH-80/PDMS $)_{15}$ film decreases by more than 3 orders of magnitude compared with that of untreated $\mathrm{Al}$ substrate. In
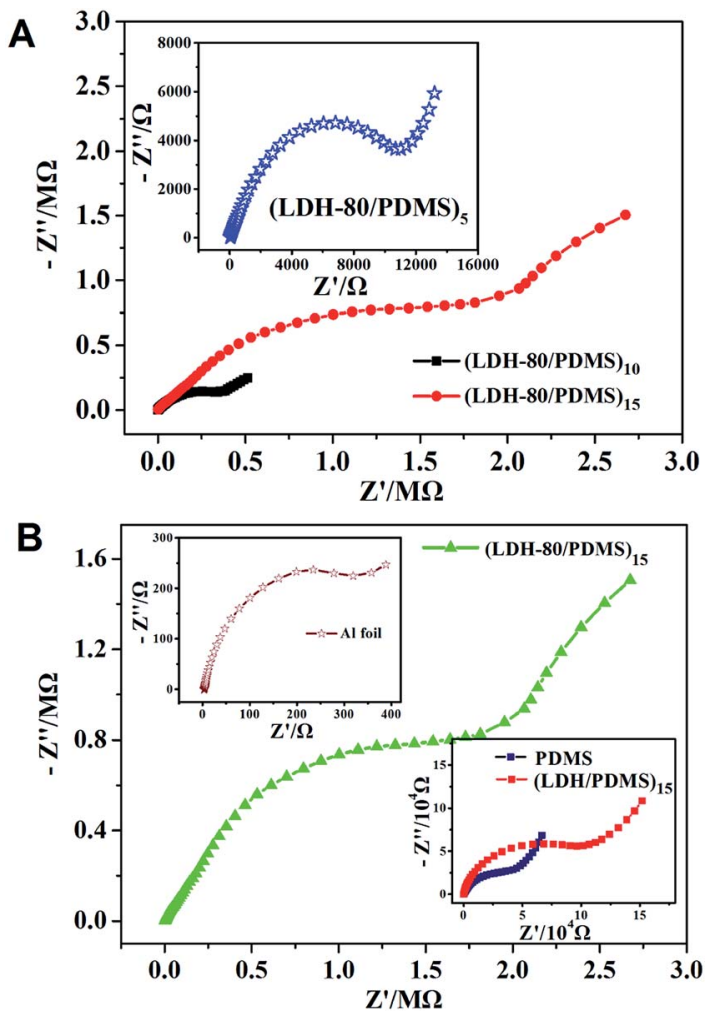

Fig. 6 (A) Nyquist plots of the impedance for (LDH-80/PDMS) ${ }_{n}$ films on Al substrate with $n=5$ (inset), 10 and 15; (B) Nyquist plots of the impedance for various samples, including (LDH-80/PDMS) ${ }_{15}$ film, bare Al substrate (top left), PDMS and (LDH/PDMS) ${ }_{15}$ film (bottom right). The red line in $A$ is the same as the green line in $B$ for giving a clear comparison.
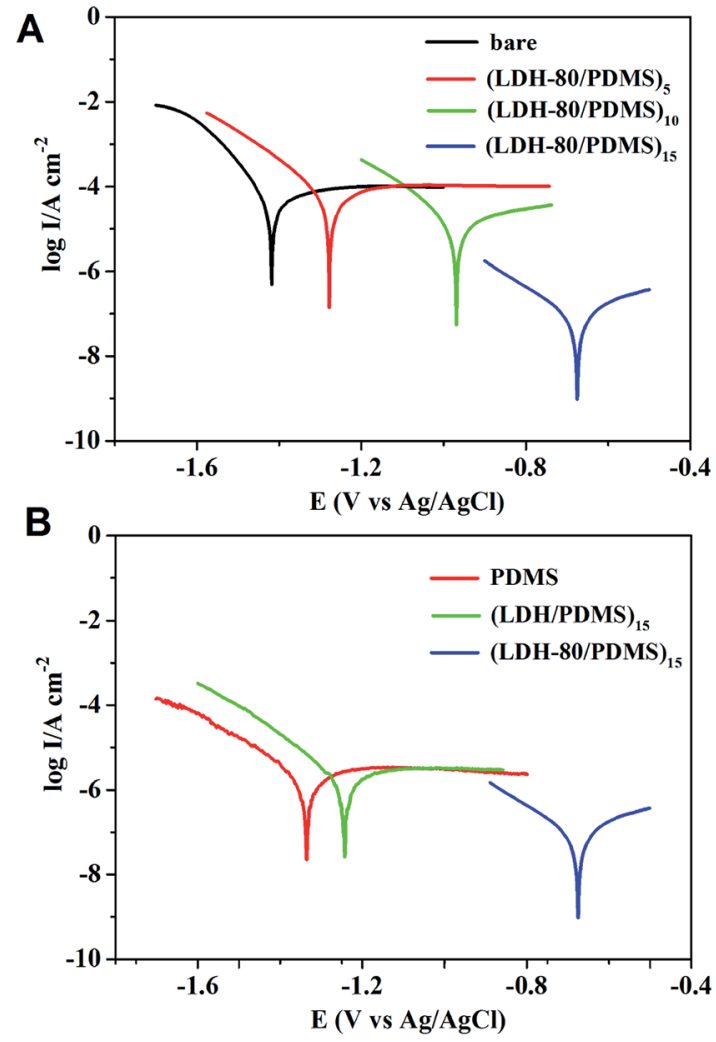

Fig. 7 (A) Potentiodynamic polarization curves of bare Al substrate, $(\mathrm{LDH}-80 / \mathrm{PDMS})_{n}$ films on Al substrate with $n=5,10$ and 15; (B) Potentiodynamic polarization curves of PDMS, (LDH/PDMS) 15 and $(\mathrm{LDH}-80 / \mathrm{PDMS})_{15}$ film, respectively.

addition, the (LDH-80/PDMS $)_{15}$ film shows a larger $E_{\text {corr }}$ and smaller $I_{\text {corr }}$, in comparison with pure PDMS and (LDH/PDMS $)_{15}$ film (Fig. 7B), indicating its better anti-corrosion effect. The Tafel extrapolation test combined with EIS measurement demonstrate that the dual-barrier property of (LDH-80/PDMS $)_{15}$ film exerts a remarkable anti-corrosion performance toward the metal surface protection.

In addition, the anti-corrosion effect of (LDH-80/PDMS) 15 film on $\mathrm{Al}$ substrates was further confirmed by SEM. The bare $\mathrm{Al}$ foil exhibits a uniform surface without any corrosion (Fig. 8A);

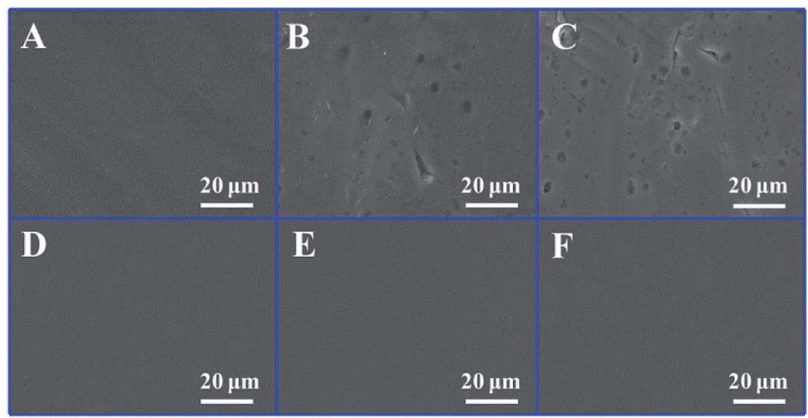

Fig. 8 Top-view SEM images of Al substrate dipping into a $3.5 \% \mathrm{NaCl}$ solution for (A) 0 day, (B) 90 days and (C) 180 days, respectively; topview SEM images of (LDH-80/PDMS) ${ }_{15}$ film coated Al substrate dipping into the same solution for (D) 0 day, (E) 90 days and (F) 180 days, respectively. 
after dipping into a $3.5 \% \mathrm{NaCl}$ solution in air for 90 days, an obvious pitting corrosion was observed on the surface (Fig. 8B); this became more serious with an extension to 180 days (Fig. 8C). In contrast, the (LDH-80/PDMS $)_{15}$ film coated $\mathrm{Al}$ substrate did not undergo obvious corrosion at the same conditions (Fig. 8D-F). The results demonstrate the potential application of (LDH-80/PDMS $)_{n}$ films in the protection of metal surface.

\section{Conclusions}

In summary, organic-inorganic (LDH-80/PDMS $)_{n}$ films are fabricated by an alternate spin-coating method, which display excellent oxygen and water vapor dual-barrier property. The enhanced $\mathrm{O}_{2}$ gas barrier property is mainly due to the elongation of diffusion path; while the water vapor barrier behavior is attributed to the synergistic effect of hydrophobicity and increased diffusion path. By virtue of such dual-barrier property, the Al substrate coated by (LDH-80/PDMS $)_{n}$ films shows a significantly improved anti-corrosion effect through restraining the oxygen-consuming corrosion. Therefore, this work provides a new strategy for developing oxygen/water vapor dualbarrier films, which are highly needed for a variety of applications, including metal anti-corrosion, food/pharmaceutical packaging and electronics encapsulation.

\section{Conflicts of interest}

There are no conflicts to declare.

\section{Acknowledgements}

This work was supported by the 973 Program (Grant No. 2014CB932104), the National Natural Science Foundation of China (NSFC), the Fundamental Research Funds for the Central Universities, the Young Elite Scientists Sponsorship Program by CAST, and the Beijing Nova program. The authors thank for the support from the SSRF (Shanghai Synchrotron Radiation Facility) during the grazing-incidence XRD measurements.

\section{Notes and references}

1 Y. Yang, L. Bolling, M. Haile and J. C. Grunlan, RSC Adv., 2012, 2, 12355-12363.

2 N. Yan, F. Capezzuto, M. Lavorgna, G. G. Buonocore, F. Tescione, H. Xia and L. Ambrosio, Nanoscale, 2016, 8, 10783-10791.

3 T. Kim, J. H. Kang, S. J. Yang, S. J. Sung, Y. S. Kim and C. R. Park, Energy Environ. Sci., 2014, 7, 3403-3411.

4 M. Wang, V. Janouta and S. L. Regen, Chem. Commun., 2013, 49, 3576-3578.

5 S. Seethamraju, P. C. Ramamurthy and G. Madras, RSC Adv., 2014, 4, 11176-11187.

6 J. Yoo, S. B. Lee, C. K. Lee, S. W. Hwang, C. Kim, T. Fujigaya, N. Nakashima and J. K. Shim, Nanoscale, 2014, 6, 1082410830 .
7 A. Mace, K. Laasonenb and A. Laaksonen, Phys. Chem. Chem. Phys., 2014, 16, 166-172.

8 C. Xiang, P. J. Cox, A. Kukovecz, B. Genorio, D. P. Hashim, Z. Yan, Z. Peng, C.-C. Hwang, G. Ruan, E. G. Samuel, P. M. Sudeep, Z. Konya, R. Vajtai, P. M. Ajayan and J. M. Tour, ACS Nano, 2013, 7, 10380-10386.

9 S. Cheng, Y. Zhang, R. Cha, J. Yang and X. Jiang, Nanoscale, 2016, 8, 973-978.

10 Y. Lin, Z. Zeng, J. Zhu, S. Chen, X. Yu and L. Liu, RSC Adv., 2015, 5, 57771-57780.

11 Y.-H. Yang, L. Bolling, M. A. Priolo and J. C. Grunlan, Adv. Mater., 2013, 25, 503-508.

12 C. Zhang, C. Zhang, R. Ding, X. Cui, J. Wang, Q. Zhang and Y. Xu, ACS Appl. Mater. Interfaces, 2016, 8, 14766-14775.

13 Y. C. Li, J. Schulz, S. Mannen, C. Delhom, B. Condon, S. Chang, M. Zammarano and J. C. Grunlan, ACS Nano, 2010, 4, 3325-3337.

14 A. J. Svagan, A. Åkesson, M. Cárdenas, S. Bulut, J. C. Knudsen, J. Risbo and D. Plackett, Biomacromolecules, 2012, 13, 397-405.

15 H. Liu, T. Kuila, N. H. Kim, B. C. Ku and J. H. Lee, J. Mater. Chem. A, 2013, 1, 3739-3746.

16 S. Zhou, J. Chen, X. Li, X. Ji, G. Zhong and Z. Li, RSC Adv., 2016, 6, 2530-2536.

17 M. A. Priolo, D. Gamboa, K. M. Holder and J. C. Grunlan, Nano Lett., 2010, 10, 4970-4974.

18 H. Huang, C. Liu, D. Li, Y. Chen, G. Zhong and Z. Li, J. Mater. Chem. A, 2014, 2, 15853-15863.

19 C. Aulin, G. S. Alvarez and T. Lindström, Nanoscale, 2012, 4, 6622-6628.

20 S. Burgess, J. Lee, C. Mubarak, R. Kriegel and W. Koros, Polymer, 2015, 65, 34-44.

21 Y. Wang, Q. Yang, J. Li, J. Yang and C. Zhong, Phys. Chem. Chem. Phys., 2016, 18, 8352-8358.

22 K. Bhunia, H. Zhang, F. Liu, B. Rasco, J. Tanga and S. Sablani, Innovative Food Sci. Emerging Technol., 2016, 38, 124-130.

23 S. Despond, E. Espuche and A. Domard, J. Polym. Sci., Part B: Polym. Phys., 2001, 39, 3114-3127.

24 L. Wu, Z. Hu, G. Chen and Z. Li, Soft Matter, 2015, 11, 90389044.

25 Y. Wang, D. Zhang, Q. Bao, J. Wu and Y. Wan, J. Mater. Chem., 2012, 22, 23106-23113.

26 G. Abellán, E. Coronado, C. Martí-Gastaldo, A. Ribera, J. L. Jordá and H. García, Adv. Mater., 2014, 26, 4156-4162.

27 Z. Gu, J. J. Atherton and Z. P. Xu, Chem. Commun., 2015, 51, 3024-3036.

28 W. Shi, Y. Jia, S. Xu, Z. Li, Y. Fu, M. Wei and S. Shi, Langmuir, 2014, 30, 12916-12922.

29 L. Yan, W. Chen, X. Zhu, L. Huang, Z. Wang, G. Zhu, V. A. L. Roy, K. N. Yu and X. Chen, Chem. Commun., 2013, 49, 10938-10940.

30 S. Sene, S. Bégu, C. Gervais, G. Renaudin, A. Mesbah, M. E. Smith, P. H. Mutin, A. van der Lee, J.-M. Nedelec, C. Bonhomme and D. Laurencin, Chem. Mater., 2015, 27, 1242-1254. 
31 T. Tsai, B. Naveen, W. Shiu and S. Lu, RSC Adv., 2014, 4, 25683-25691.

32 Y. Dou, S. Xu, X. Liu, J. Han, H. Yan, M. Wei, D. G. Evans and X. Duan, Adv. Funct. Mater., 2014, 24, 514-521.

33 Y. Dou, T. Pan, S. Xu, H. Yan, J. Han, M. Wei, D. G. Evans and X. Duan, Angew. Chem., Int. Ed., 2015, 54, 9673-9678.

34 T. Pan, S. Xu, Y. Dou, X. Liu, Z. Li, J. Han, H. Yan and M. Wei, J. Mater. Chem. A, 2015, 3, 12350-12356.
35 Y. Dou, A. Zhou, T. Pan, J. Han, M. Wei, D. Evans and X. Duan, Chem. Commun., 2014, 50, 7136-7138.

36 J. Han, Y. Dou, M. Wei, D. G. Evans and X. Duan, Angew. Chem., Int. Ed., 2010, 49, 2171-2174.

37 Y. Dou, J. Han, T. Wang, M. Wei, D. G. Evans and X. Duan, Langmuir, 2012, 28, 9535-9542. 